\title{
Dynamics of laser-excited nanoparticles
}

\author{
Anton Plech ${ }^{a}$, Andreas Siems ${ }^{a}$, Vassilios Kotaidis $^{a}$ and Andreas Menzel $^{b}$ \\ ${ }^{a}$ Department of Physics and Center for Applied Photonics, University Konstanz, \\ D-78457 Konstanz, EU. \\ ${ }^{b}$ Paul Scherrer Institut, CH-5232 Villigen PSI, Switzerland.
}

\begin{abstract}
By the use of stroboscopic laser pump - x-ray probe techniques and x-ray scanning techniques the structural relaxations of gold nanoparticles have been resolved on the $50 \mathrm{ps}$ time scale. The structural dynamics are addressed by several methods including powder scattering and small angle scattering (SAXS) to resolve microscopic and mesoscopic length scales of the composite system. The laser power is a direct measure of the dissipated heat. Thus the caloric reaction and melting transition can be monitored as function of temperature, particle size and time. Nonlinear effects are observed with femtosecond excitation, attributed to ablation. While the phenomenology for nanoparticle suspensions and surface supported monolayers display similar energetics, structure formation processes are strongly altered on the surface due to the interparticle interactions.
\end{abstract}

Keywords: laser irradiation, gold nanoparticles, x-ray scattering, structural dynamics

\section{INTRODUCTION}

Transition metal nanoparticles receive large attention in recent research. Their optical properties can be tuned by means of size and shape manipulation or geometrical arrangement. ${ }^{1}$ Applications in photonic science include plasmon waveguides or substrates for surface-enhanced Raman scattering (SERS). In biological applications the ability to form hybrids with biomolecules for sensing and tissue interaction are appealing qualities. The strong interaction with visible light allows for sensing weak interactions of the nanomaterial and on the other hand also for the manipulation of the nanomaterial by intense laser light. The linear and nonlinear reactions of nanoparticles with laser light have been clarified in a number of studies. They revealed the nature of the interaction with the free-electron gas, lattice heating, ${ }^{2}$ heat dissipation, ${ }^{3-5}$ or melting. ${ }^{6-9}$

While reversible relaxations are quite well understood, non reversible reactions of nanoparticles show a variety of phenomena, ${ }^{10,11}$ where much less is understood in terms of mechanisms. The phenomenology of ablation of surfaces by short laser pulses is well understood and utilized for particle synthesis. ${ }^{12}$ The interaction of laser light with particles may cause similar phenomena, allowing sub-wavelength structuring of nanoscale materials by lasers pulses. ${ }^{13,14}$ The shape related optical resonances result in anisotropic structure modifications. We have recently clarified an anisotropic ablation on suspended gold particles induced by the dipolar near-field enhancement due the electron gas excitation. ${ }^{15}$ This has been interpreted as optical rectification at the particle surface by Vella et al. ${ }^{16}$ Further evidence of the nonlinear near field ablation effect has been given by the formation of sub-wavelength ablation patterns near triangular particles on a surface. ${ }^{17}$ In a recent optical study the structure of irradiated silver particles has been studied and revealed different mechanisms of particle modification by weak multiple excitation in contrast to intense single-shot excitation. ${ }^{18}$

A clarification of a structural mechanism should include the knowledge of the relative importance of dissipation channels of the applied light, the structural relaxations and effects of the particle size and morphology. As the intrinsic dynamics of electron and phonon interactions enroll on very short time scales one needs ultrafast methods to follow the reactions directly. Within this report we show that, by applying picosecond pump probe schemes with x-rays as probe pulses ${ }^{19,20}$ as well as steady state post-mortem analysis by x-ray scattering methods, the relaxations in gold nanoparticle materials can be rationalized and interpreted in terms of thermal or non-thermal reactions.

Send correspondence to A.P.: E-mail: anton.plech@uni-konstanz.de

Photon Processing in Microelectronics and Photonics VII, edited by Andrew S. Holmes, Michel Meunier, Craig B. Arnold, Hiroyuki Niino, David B. Geohegan, Frank Träger, Jan J. Dubowski, Proc. of SPIE Vol. 6879, 68790H, (2008) $\cdot 0277-786$ X/08/\$18 $\cdot$ doi: $10.1117 / 12.774664$ 


\section{MATERIALS AND METHODS}

\subsection{Gold particles and monolayers}

Gold nanoparticles have been produced by the widespread synthesis procedure as described by Turkevich. ${ }^{21,22}$ The reduction of a diluted solution of hydrochloro aurate $(1-2 \mathrm{mM}$, Chempur) by a defined amount of sodium tris citrate (Merck) at the boiling point of water yields fairly monodispersed gold particles, that are stabilized by the electrostatic double layer of the adsorbed citrate species. The size is defined by the ratio of gold versus citrate ions and can be tuned within a diameter range of 10-100 nm. The suspension can be used as synthesized for the laser pump -x-ray probe experiment as described in section 2.2. As the particle concentration is typically a few nanomolar the distances between particles are much larger than their diameter. Therefore the particles in the suspensions can be regarded as isolated, simplifying the interpretation of the results.

Particle materials can be generated by adsorbing the synthesized particles onto a flat surface. By preassembly of either a polyelectrolyte ${ }^{23}$ or a aminothiol layer onto a glass ${ }^{24}$ or silicon surface, one obtains a sub-monolayer coverage of particles. The particles are distributed randomly on the surface. The coverage of below $10 \%$ still results in a high probability of touching particles. The extinction color therefore changes from the native ruby red to a violet red to blue. The plasmon resonance of the bare particles around $524 \mathrm{~nm}$ (of the $28 \mathrm{~nm}$ particles used herein), $528 \mathrm{~nm}$ (38 nm particles) to $537 \mathrm{~nm}$ ( $55 \mathrm{~nm}$ particles) shifts due to the electromagnetic coupling towards 600 to $650 \mathrm{~nm} \cdot{ }^{23}$ However, when exciting either the suspension or the adsorbed particles with UV light $(400 \mathrm{~nm})$ the extinction and, consequently the light absorption, is largely unaffected by the relative arrangement.

\subsection{Time-resolved x-ray scattering}

The general idea of time-resolved x-ray scattering is to synchronize an excitation source, such as a femtosecond laser, with a source of pulsed x-rays. Then a sequence of temporally defined pulse pairs excite, or probe the sample, respectively, in a stroboscopic manner. In our case the $100 \mathrm{ps}$ x-ray pulses emitted from the storage ring ESRF (beam station ID09B) are isolated at $1 \mathrm{kHz}$ repetition rate and synchronized with a femtosecond oscillator as input for a multi pass amplifier (KMLabs). The details of the setup can be found elsewhere. ${ }^{19,25}$ The important point is, that the $\mathrm{x}$-ray pulses are very intense, so that scattering patterns with high signal to noise contrast can be obtained even for disordered materials with a small number of repetitions. The quality of the synchrotron beam allows to employ different scattering processes as in conventional x-ray scattering, such as powder scattering, ${ }^{7}$ small angle scattering SAXS, ${ }^{20}$ or liquid scattering. ${ }^{26}$ These methods allow to investigate the crystallinity, shape of nanoparticles, as well as the reactions of the medium.

The beams are either focussed onto a thin-walled glass capillary (Hilgenberg, $10 \mu \mathrm{m}$ wall thickness), in which the gold nanoparticle suspension is circulated, or on a thin glass wafer (microscope cover slides), with a sub-monolayer coverage of particles. The x-ray scattering pattern is collected on a two dimensional $\mathrm{x}$-ray camera (charge-coupled device with scintillator detection, MarCCD, diameter $133 \mathrm{~mm}$ ) which is put at appropriate distances (630 mm after the sample for SAXS at $15 \mathrm{keV} ; 140 \mathrm{~mm}$ for powder scattering of the gold (111) powder ring at $15 \mathrm{keV}$ ).

As the typical change of the diffraction pattern as induced by laser excitation is very small (in the range of $10^{-3}$ to $10^{-2}$ ). the differences between excited and ground state are computed and used for the interpretation of the SAXS signal. Thereby any parasitic scattering, which is not related to the photo-excitation cancels out. In powder scattering the peak position as function of scattering vector $\mathrm{Q}=4 \pi / \lambda \cdot \sin (2 \Theta / 2)$ ( $\mathrm{x}$-ray wavelength $\lambda$ and scattering angle $2 \Theta$ ) and the peak shape can be utilized to derive lattice temperature and strain. In general, lattice heating and dynamic strain can not be unambiguously discerned (at least with only one or two Bragg reflections), but in the present case the coherent lattice excitation only amounts for a small fraction of the observed dynamics, ${ }^{27}$ so that an interpretation of peak shift as lattice heating is reasonable.

SAXS can be interpreted in terms of shape changes of the particles. The description of an ensemble of spherical particles can be given by analytical formulae, which show a strong scattering $I(Q)$ in forward 
direction with an angular extension around the direct beam $(Q=0)$ of the order of $1 / \mathrm{R}$ of the radius of the particles. For larger $Q$ the scattering intensity decays with $Q^{-4}$ with intermittent size related oscillations. Importantly, the intensity in forward direction is a measure for the particle volume $V{ }^{28}$ which is weighted by the scattering length density $\Delta \rho(\vec{r})$.

$$
\begin{aligned}
I(Q) & \propto I_{0}\left|\int_{V} \Delta \rho(\vec{r}) \exp (i Q \vec{r}) d \vec{r}\right|^{2} \\
& =I_{0} \times S(Q) \\
& \propto V^{2}(\Delta \rho)^{2} \times F(Q, R)^{2},
\end{aligned}
$$

with the particle form factor of a homogeneous sphere with radius $\mathrm{R}$ :

$$
F(Q, R)=3[\sin (Q R)-(Q R) \cdot \cos (Q R)] /(Q R)^{3} .
$$

For an ellipsoidal object in particular, the anisotropic SAXS formulae can be generalized by using the scattering vectors along the long and short axis of the ellipsoid $Q_{y}$ and $Q_{z} \cdot{ }^{20,28}$ This is done by replacing $Q \cdot R$ in eq. 4 by

$$
X\left(Q_{y}, Q_{z}\right)=\sqrt{Q_{y}^{2} R^{2}+Q_{z}^{2}(\chi R)^{2}}
$$

with $\chi$ being the relative change of the shorter axis of an oblate rotational ellipsoid relative to the longer axis. The $\mathrm{x}$-ray beam propagates in $x$ direction.

\subsection{Scanning $\mathrm{X}$-ray analysis}

As will be shown in the next section, the application of laser pulses on a nanoparticle covered surface will cause irreversible changes of the particles, which can be analyzed post-mortem by optical methods ${ }^{13,14,18}$ or x-ray scattering methods. The general idea is to irradiate the surface with a laser shot of defined fluence with, say, a Gaussian intensity distribution. If the damage is a function of local fluence, then the damage pattern will display a spatial modulation according to the focus size of the laser beam. We have irradiated the sample with a single shot from a femtosecond laser (Spectra Physics Spitfire, seeded by Spectra Physics Tsunami) with a focus size of $390 \mu \mathrm{m}$ (full width at half maximum) at $400 \mathrm{~nm}$ wavelength.

In order to characterize the irradiation products we used spatially resolved SAXS at the Swiss Light Source, cSAXS beamline. An x-ray beam with a footprint of about $15 \mu \mathrm{m}$ was scanned over the sample, and SAXS patterns were recorded with the PILATUS $2 \mathrm{M}$ detector. ${ }^{29}$ Since the laser beam profile (is well characterized each position, at which the SAXS intensity distribution was recorded, can directly be related to a specific laser fluence, despite any non-local effects or material transport.

\section{RESULTS AND DISCUSSION}

A precise knowledge of the thermal kinetics of laser excited particles is necessary to gain information about the mechanisms of structuring of the nanoparticle material. The relaxation times for heating, melting and cooling are in the range of some picoseconds (electron phonon coupling ${ }^{30}$ ) or hundreds of picosecond for the cooling of particles in aqueous suspension ${ }^{7,31}$ to tens of nanoseconds for supported particles on a surface. ${ }^{27}$ Therefore the lattice expansion was measured after the shortest accessible time delay after the laser excitation. This is in the range of 50-80ps for a 100ps laser pulse. Although the expansion generally shows lattice strain from non-thermal motion,${ }^{27}$ the main contribution nevertheless can be attributed to thermal expansion. Thereby the particle temperature can be deduced quite reliably. In fig. 1 a) and c) the expansion of two different samples are compared, the first with a particle suspension, the second with adsorbed particles. In both cases the rise of the expansion versus laser fluence starts linearly with a 
similar slope. This linearity shows that laser light absorption is a linear process. While both the latent heat and the expansion coefficient are weakly dependent on temperature, both effects are opposed to each other. Therefore only a small deviation from linearity is expected. Secondly the extinction at the interband absorption of gold is similar for both particles in first place.

At higher fluence the behavior of the lattice expansion changes. For the suspended particles (fig. 1a)) the expansion reaches almost the value of the bulk melting point (1.78\%), after which the Bragg scattering disappears, which is indicative of particle melting. For the adsorbed particles the expansion levels off at considerable lower expansion, from where on it remains almost constant as function of fluence. The general difference of both experiments is that the adsorbed particles were excited repetitively, while a true single-shot excitation was possible for the suspended particles, which were flushed through the interaction point at sufficient speed. However, when exposing the particles on the surface to single shots with appropriate sample displacement, one can indeed recover the maximum expansion near the melting point. The present observation shows that the particles degrade by repetitive excitation, when passing some fluence limits. ${ }^{32}$ This fluence limit of about $90 \mathrm{~J} / \mathrm{m}^{2}$ corresponds to a lattice heating of about $650 \mathrm{~K}$ which is still below the particle melting point. Despite the complications of multiple shot excitation the presented lattice expansion of the supported particles allows to determine the melting threshold fluence, by extrapolating the initial slope towards the maximum possible lattice expansion. This can be located here at about $140 \mathrm{~J} / \mathrm{m}^{2}$.

The changes of diffraction intensity in the SAXS region due to the laser excitation are depicted in fig. 2. The data are derived by subtracting the scattering intensity of the native sample from the intensity after laser excitation. In the case of the post-mortem analysis, the reference was taken far away from the laser spot. The spatial coordinates of the images mark the scattering vector $Q_{y}$ and $Q_{z}$ in horizontal and vertical direction relative to the direct beam path, respectively. They can directly be interpreted in terms of morphological changes of the particles in these two spatial directions. The particles in this study are almost spherical and moreover randomly distributed in the suspension and on the support. Therefore the scattering from the native sample displays a spherical symmetry. In all of the selected laser irradiation images in fig. 2 the patterns lack the rotational symmetry of the scattering from isotropic samples without laser excitation.

The scattering anisotropy after laser excitation is given by a polar axis, which is vertical and coincides with the laser beam polarization. For the suspended particles a time delay of 2 ns was chosen, because for shorter delays one observes transient structural response of the surrounding water, which forms vapor bubbles around the gold particles ${ }^{33}$ upon strong superheating. These vapor bubbles induce a strong modification of the SAXS signal. However, a slight anisotropy is already visible for short delays. This becomes clearer for the particles on the surface, where the pattern is fully developed within the first 100 ps. In solution the anisotropy disappears only after some microseconds after rotational diffusion of the particles. On the surface the particles tend to be fixed, so that the observed anisotropy remains stationary. This can be seen by the post-mortem analysis in the right image of fig. 2 of the sample 4 days after the irradiation.

Despite some similarities, such as a defined axis along the laser polarization, the scattering of the suspended and the adsorbed particles differs fundamentally. For the suspended particles the scattering in the forward direction ( $Q \rightarrow 0$, center of the images) is decreased, while for the other images the scattering increases strongly in the forward direction. Consequently, the particle shape modifications are different in both cases. The reduction of scattering in the first case is described by a volume reduction of the particles, which has been reported as material ablation from the surfaces at the two poles. ${ }^{15}$ At the same time, at finite $Q_{z}$ the scattering increases, as the new form factor in that direction falls off at a higher $\mathrm{Q}$, relative to the form factor of the native particles. Due to reciprocity the real space length scale in this direction decreases. In the horizontal plane no strong changes occur, pointing towards a preserved size. This fits the description of volume loss only at the poles of the spheres.

For the surface-adsorbed particles the intensity increases in the forward direction $\left(\mathrm{Q}<0.01 \AA^{-1}\right)$, which, for isolated particles, would be interpreted as an increase in volume. This would be astounding. One should, however, recapitulate that in our case the particles are organized in one layer and stay in close 

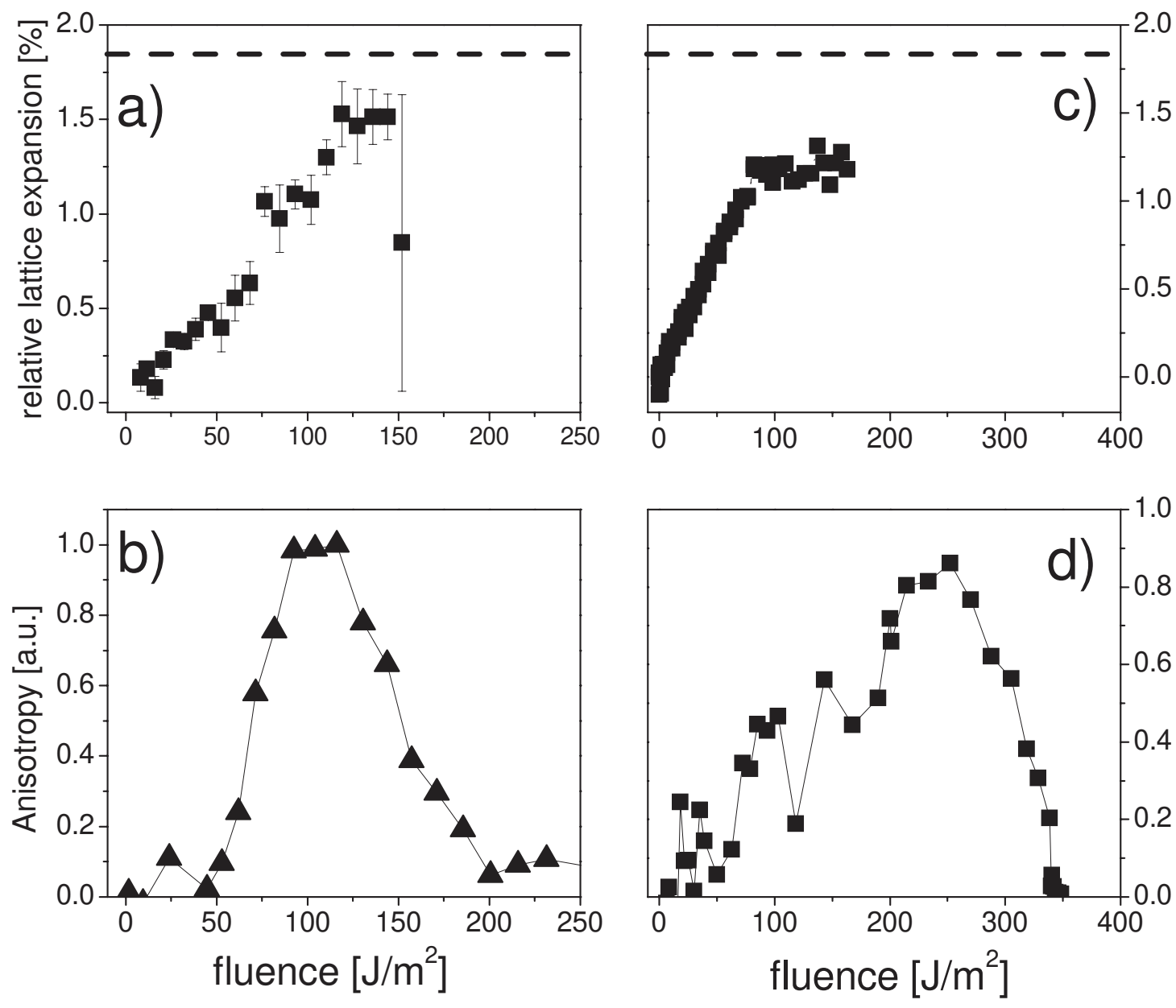

Figure 1. Lattice expansion of gold particles after laser excitation and anisotropy of the SAXS scattering pattern as function of laser fluence. a) Lattice expansion of $38 \mathrm{~nm}$ gold particles in aqueous suspension at a delay of 80 ps. b) Corresponding SAXS anisotropy as an indicator of anisotropic ablation. c) Expansion of gold particles of $56 \mathrm{~nm}$ as sub-monolayer coverage on a glass wafer at $80 \mathrm{ps}$ delay. The dashed lines in a) and c) mark the maximum expansion of bulk gold at the melting point. d) Anisotropy of the SAXS pattern of the sample after single shot excitation in c) in a scanning post-mortem analysis. The x-ray footprint was scanned across the laser footprint and the spatial axis has been mapped to a fluence dependence. 


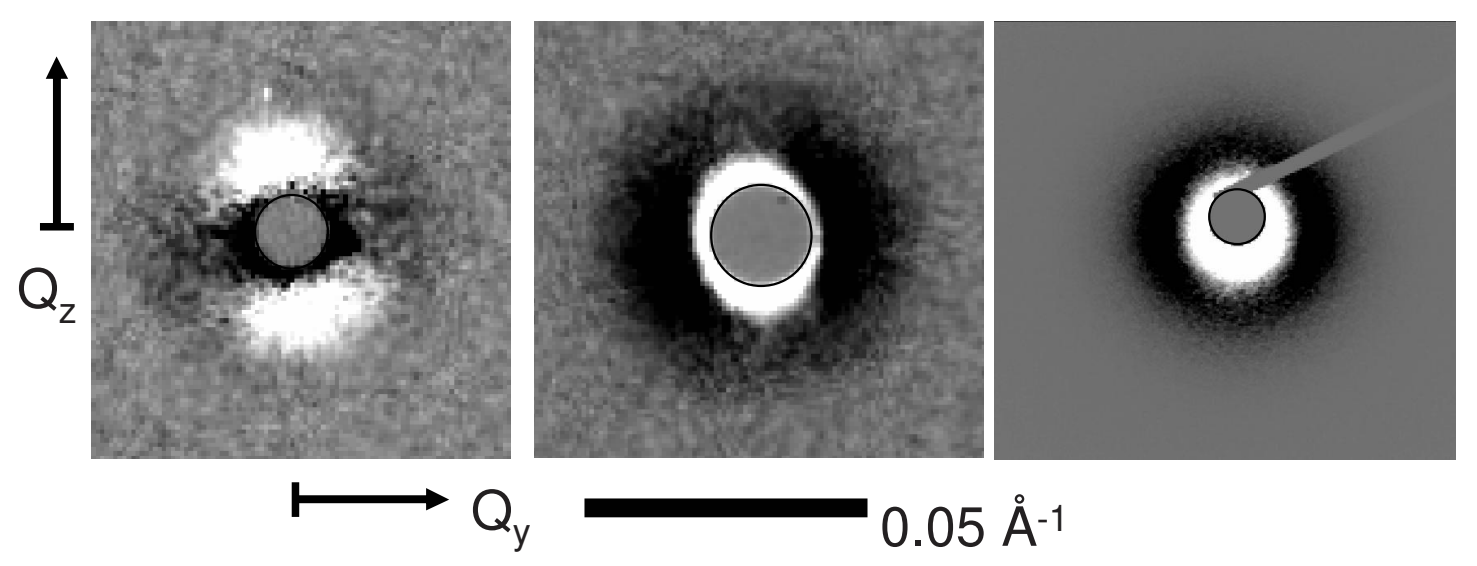

Figure 2. Difference scattering intensity from laser-excited samples containing gold particles relative to the nonirradiated sample. The changes are plotted in grey scale with intensity increase as bight areas and intensity decrease as dark areas. Grey marks unchanged areas. The laser polarization in all cases is aligned almost vertically. From left: Pattern of gold particles of $38 \mathrm{~nm}$ in suspension at $2 \mathrm{~ns}$ delay excited with $120 \mathrm{~J} / \mathrm{m}^{2}$; gold particles of $28 \mathrm{~nm}$ on a glass wafer surface at $100 \mathrm{ps}$ delay at a fluence of $220 \mathrm{~J} / \mathrm{m}^{2}$; gold particles of $55 \mathrm{~nm}$ on a glass wafer in a scanning post mortem analysis at a fluence of $230 \mathrm{~J} / \mathrm{m}^{2}$. The central hatched area is masked by the direct-beam block.

contact. As a consequence the single particle scattering is modified by the position correlation function of particle pairs. This modifies the SAXS intensity such that the intensity is reduced in forward direction for the native sample, while excess scattering is observed, where the scattering vector equals the inverse distance of adjacent particles. This is shown in fig. 3 with two azimuthally averaged scattering curves of the native sample and the laser irradiated portion. The difference in particular between the curve from the native sample and the calculation of a sphere form factor according to eq. 4 shows this modification. With laser excitation two things can happen: the particles can be moved against each other while ablation occurs. This at the same time changes the sizes and destroys the positional correlation. Furthermore, at elevated excitation the particles can also start to fuse together. This fusing predominantly happens near the melting temperature, but can be observed for continuous heating already at very moderate temperatures. ${ }^{34}$ The consequence is in both cases a restoration of the scattering intensity in the forward direction, be it due to suppression of positional particle modifications, be it because of fused particles. These modifications are anisotropic, as evidenced by fig. 2. Therefore the positional changes and the laser fusion of the particles are anisotropic. The driving force is again the near-field ablation at the two poles of the particles. This induces a net force along the laser polarization to squeeze the product together, starting from inhomogeneities, while the lateral size merely increases.

When regarding the fluence dependence of this effect one can observe interesting threshold phenomena. For the particles in aqueous suspension the picture is quite clear. The SAXS anisotropy is shown in fig. $1 \mathrm{~b}$ ) for a series of measurements with varying fluence. The signal starts in a fluence range where the particles are solid and is suppressed when the fluence enters the melting region. Only as long as it is solid the particle can preserve anisotropic ablation. Once it melts it relaxes back into a spherical shape, which restores the isotropic scattering distribution.

The anisotropy has been measured for the adsorbed particles in the post-mortem analysis as a function of position across the laser spot, i.e. as function of laser fluence. Here the anisotropy also starts below the melting region of the particles, while it is maintained even for molten particles. As the signal is caused mostly by the modification of the positional correlation of particles including particle fusion, the melting transition does not represent a threshold for the observation of shape anisotropy. The signal vanishes quickly above $320 \mathrm{~J} / \mathrm{m}^{2}$, which, however, does not relate to a symmetric relaxation, but rather a loss of particles from the surface. Indeed it can be seen, that the center of the spot is completely clean, as well as the SAXS intensity drops drastically. This is a well known phenomenon, called laser cleaning. Laser 


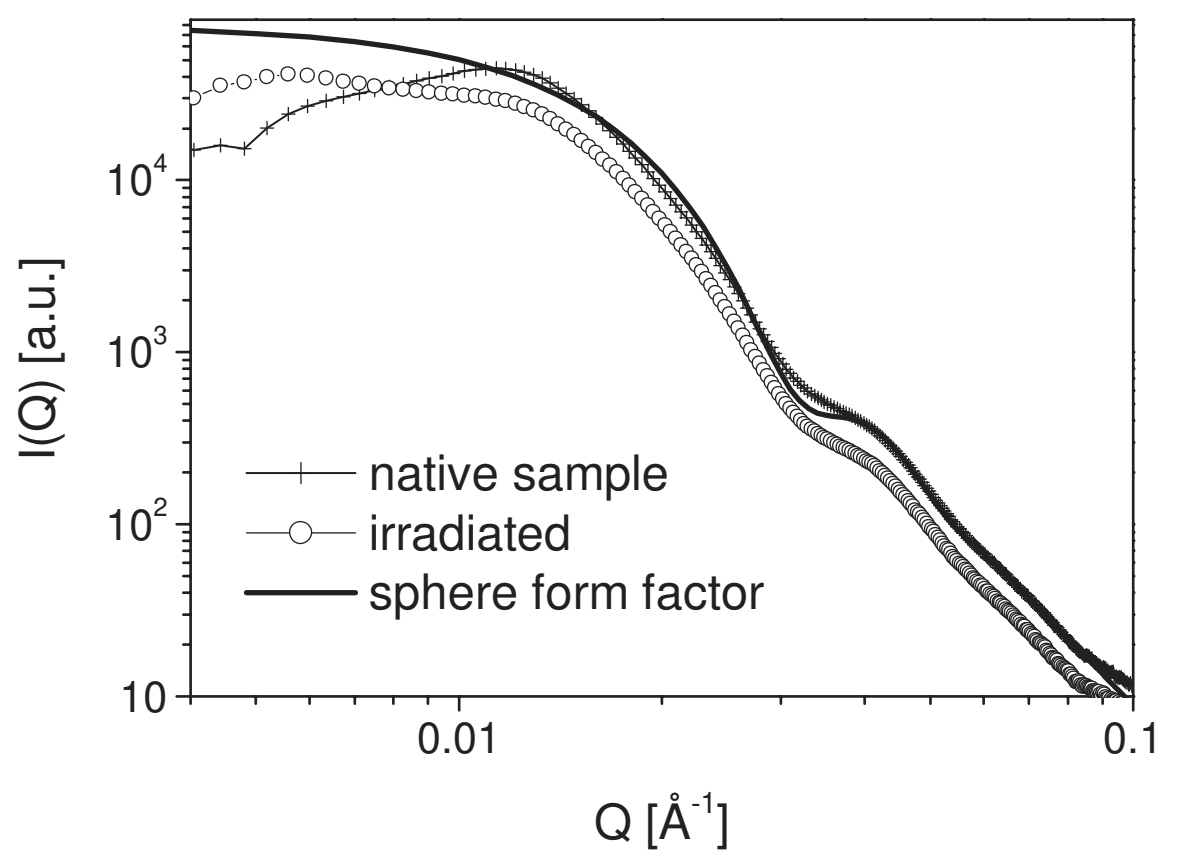

Figure 3. SAXS intensity of gold particles on a glass surface without laser excitation (native state) and near the center of the irradiated spot together withe a simulation of a sphere according to eq. 4. The scattering from the native sample peaks around $\mathrm{Q}=0.013 \AA^{-} 1$, while the intensity is reduced for lower $\mathrm{Q}$ due to the positional correlations of the particles.

cleaning can either be initiated by particle dynamics ${ }^{35}$ (dry laser cleaning) or matrix related expulsion (steam or matrix laser cleaning), ${ }^{36}$ respectively.

\section{CONCLUSION}

As a result the femtosecond laser excitation of metal nanoparticles has several facets. The thermal history can be addressed conveniently by powder scattering, where the Bragg peak shift relates to the particle temperature as function of delay after the laser excitation. The loss of Bragg intensity directly pinpoints the melting transition. The thermal effects are shown to be easily understood by continuum calculations ${ }^{5,37}$ and reflect the fact, that nanoparticles with sizes far above $10 \mathrm{~nm}$ can be described in many aspects as bulk-like (expansion coefficient, latent heat). The ultrashort excitation with high peak electrical fields activates as well non-thermal channels such as the near-field ablation. These non-thermal effects are not primarily visible in heating and melting but rather as morphological modifications of the particles. These modifications can be observed with SAXS, which quantifies both the size change of individual particles, and the positional correlation between different particles. Both of these effects cause what can be observed macroscopically as sample degradation. The study shows that subtle effects can occur, which would then manifest themselves for example in the modified optical properties of the sample. Thus spectral changes can be induced or as well dichroitic behavior. ${ }^{14,38}$

The remarkable low thresholds for particle manipulation open new pathways for deterministic structuring on the nanoscale with macroscopic methods.

\section{ACKNOWLEDGMENTS}

We wish to thank M. Wulff, J. Boneberg, F. Pfeiffer, M. Cammarata and F. Ewald for discussions and help with the experiments. The European Synchrotron Radiation Facility and the Swiss Light Source are acknowledged for the supply of beamtime. We would like to acknowledge the funding from the German 
Science Foundation, the Ministry of Science and the Arts of Baden-Württemberg through the Center for Applied Photonics and the support from the Center for Junior Research Fellows, Konstanz.

\section{REFERENCES}

1. U. Kreibig and M.Vollmer, Optical Properties of Metal Clusters, Springer, Berlin, 1995.

2. G. V. Hartland, M. Hu, and J. E. Sader, "Softening of the symmetric breathing mode in gold particles by laser-induced heating," J. Phys. Chem. B 107, p. 7472, 2003.

3. O. M. Wilson, X. Hu, D. G. Cahill, , and P. V. Braun, "Colloidal metal particles as probes of nanoscale thermal transport in fluids," Phys. Rev. B 66, p. 224301, 2002.

4. Z. Ge, D. G. Cahill, and P. V. Braun, "Au-pd metal nanoparticles as probes of nanoscale thermal transport in aqueous solution," J. Phys. Chem. B 108, p. 18870, 2004.

5. V. Kotaidis, C. Dahmen, G. von Plessen, F. Springer, and A. Plech, "Excitation of nanoscale vapor bubbles at the surface of gold nanoparticles in water," The Journal of Chemical Physics 124(18), p. $184702,2006$.

6. S. Link and M. A. El-Sayed, "Spectroscopic determination of the melting energy of a gold nanorod," J. Chem. Phys. B 114, p. 2362, 2001.

7. A. Plech, V. Kotaidis, S. Grésillon, C. Dahmen, and G. von Plessen, "Laser-induced heating and melting of gold nanoparticles studied by time-resolved x-ray scattering," Phys. Rev. B 70, p. 195423, 2004.

8. Inasawa, M. Sugiyama, and Y. Yamaguchi, "Laser-induced shape transformation of gold nanoparticles below the melting point: The effect of surface melting," J. Phys. Chem. B 109, p. 9404, 2005.

9. H. Petrova, J. P. Juste, I. Pastoriza-Santos, G. V. Hartland, L. M. Liz-Marzán, and P. Mulvaney, "On the temperature stability of gold nanorods: comparison between thermal and ultrafast laser-induced heating," Phys. Chem. Chem. Phys. 8, p. 814, 2006.

10. A. V. Kabashin and M. Meunier, "Synthesis of colloidal nanoparticles during femtosecond laser ablation of gold in water," J. Appl. Phys. 94, p. 7941, 2003.

11. F. Stietz, "Laser manipulation of the size and shape of supported nanoparticles," Appl. Phys. A 72, p. 381, 2001.

12. F. Mafune, J. Kohno, Y. Takeda, and T. Kondow, "Full physical preparation of size-selected gold nanoparticles in solution: Laser ablation and laser-induced size control," J. Phys. Chem. B 106, p. $7575,2002$.

13. M. Kaempfe, T. Rainer, K. J. Berg, G. Seifert, and H. Graener, "Ultrashort laser pulse induced deformation of silver nanoparticles in glass," Appl. Phys. Lett. 74, p. 1200, 1999.

14. A. Podlipensky, A. Abdolvand, G. Seifert, and H. Graener, "Femtosecond laser assisted production of dichroitic 3d structures in composite glass containing ag nanoparticles," Appl. Phys. A 80, p. 1647, 2005.

15. A. Plech, V. Kotaidis, M. Lorenc, and J. Boneberg, "Femtosecond laser near-field ablation from gold nanoparticles," Nature Phys. 2, p. 44, 2006.

16. A. Vella, B. Deconihout, L. Marrucci, and E. Santamato, "Femtosecond field ion emission by surface optical rectification," Phys. Rev. Lett. 99, p. 046103, 2007.

17. P. Leiderer, C. Bartels, J. König-Birk, M. Mosbacher, and J. Boneberg, "Imaging optical near-fields of nanostructures," Appl. Phys. Lett. 85, p. 5370, 2004.

18. A. Stalmashonak, G. Seifert, and H. Graener, "Optical three-dimensional shape analysis of metallic nanoparticles after laser-induced deformation," Opt. Lett. 32, p. 3215, 2007.

19. F. Schotte, S. Techert, P. A. Anfinrud, V. Srajer, K. Moffat, and M. Wulff, Third-Generation Hard X-ray Synchrotron Radiation Sources. John Wiley and Sons, Inc., Berlin, 2002.

20. A. Plech, V. Kotaidis, K. Istomin, and M. Wulff, "Small angle pump-probe studies of photoexcited nanoparticles," J. Synchr. Rad. 90, p. 022905, 2007.

21. J. Turkevich, P. C. Stevenson, and J. Hillier, "A study of the nucleation and growth processes in the synthesis of colloidal gold," Discuss. Faraday Soc. 11, p. 55, 1951. 
22. J. Kimling, M. Maier, B. Okenve, V. Kotaidis, H. Ballot, and A. Plech, "Turkevich method for gold nanoparticle synthesis revisited," J. of Phys. Chem. B 110(32), pp. 15700-15707, 2006.

23. J. Schmitt, P. Mächtle, D. Eck, H. Möhwald, and C. A. Helm, "Preparation and optical properties of colloidal gold monolayers," Langmuir 15, p. 3256, 1999.

24. T. Sato, D. Brown, and B. F. G. Johnson, "Nucleation and growth of nano-gold colloidal lattices," Chem. Comm., p. 1007, 1997.

25. M. Wulff, A. Plech, L. Eybert, R. Randler, F. Schotte, and P. Anfinrud, "The realization of subnanosecond pump and probe experiments at the ESRF," Faraday Discussions 122, p. 13, 2003.

26. A. Plech, V. Kotaidis, M. Lorenc, and M. Wulff, "Thermal dynamics in laser excited metal nanoparticles," Chem. Phys. Lett. 401, p. 565, 2005.

27. A. Plech, S. Grésillon, G. von Plessen, K. Scheidt, and G. Naylor, "Structural kinetics of laser-excited metal nanoparticles supported on a surface," Chem. Phys. 299, p. 183, 2004.

28. A. Guinier and G. Fournet, Small-angle scattering of X-rays, John Wiley \& Sons, New York., 1955.

29. C. Broennimann, E. F. Eikenberry, B. Henrich, R. Horisberger, G. Huelsen, E. Pohl, B. Schmitt, C. Schulze-Briese, M. Suzuki, T. Tomizaki, H. Toyokawa, and A. Wagner, "The PILATUS 1M detector," Journal of Synchrotron Radiation 13, p. 120, 2006.

30. A. Arbouet, C. Voisin, D. Christofilos, P. Langot, N. D. Fatti, F. Vallée, J. Lermé, G. Celep, E. Cottancin, M. Gaudry, M. Pellarin, M. Broyer, M. Maillard, M. P. Pileni, and M. Treguer, "Electron-phonon scattering in metal clusters," Phys. Rev. Lett. 90, p. 177401, Apr 2003.

31. M. Hu and G. V. Hartland, "Heat dissipation for au particles in aqueous solution: Relaxation time versus size," J. of Phys. Chem. B 106(28), p. 7029, 2002.

32. A. Plech, V. Kotaidis, M. Wulff, C. Dahmen, and G. von Plessen, "Light-induced structural phase diagram of metal nanoparticle sols," J. Phys. Conf. Proc. 21, p. 50, 2005.

33. V. Kotaidis and A. Plech, "Cavitation dynamics on the nanoscale," Appl. Phys. Lett. 87, p. 213102, 2005.

34. A. Plech, R. Cerna, V. Kotaidis, F. Hudert, A. Bartels, and T. Dekorsy, "A surface phase transition of supported gold nanoparticles," Nano Lett. 7, p. 1026, 2007.

35. A. Habenicht, M. Olapinski, F. Burmeister, P. Leiderer, and J. Boneberg, "Jumping nanodroplets," Science 309, p. 2043, 2005.

36. B. Lukyanchuk, Laser Cleaning, World Scientific, London, 2002.

37. A. Plech, M. Wulff, S. Kuerbitz, K.-J. Berg, G. Berg, H. Graener, S. Grésillon, M. Kaempfe, J. Feldmann, and G. von Plessen, "Time-resolved x-ray diffraction on laser excited metal nanoparticles," Europhys. Lett. 61, p. 762, 2003.

38. M. Kaempfe, H. Graener, A. Kiesow, and A. Heilmann, "Formation of metal particle nanowires induced by ultrashort laser pulses," Appl. Phys. Lett. 79, p. 1876, 2001. 